\title{
Why such long faces? A response to Eugene E. Harris
}

Mark Collard ${ }^{\mathrm{a}},{ }^{*}$ and Paul O'Higgins ${ }^{\mathrm{b}}$

${ }^{a}$ Department of Anthropology and AHRB Centre for the Evolutionary Analysis of Cultural
Behaviour, University College London, London WC1E 6BT, UK

${ }^{\mathrm{b}}$ Evolutionary Anatomy Unit, Department of Anatomy and Developmental Biology, University College London, London WC1E 6BT, UK

${ }^{*}$ Correspondence (email: $\underline{\text { m.collard@ucl.ac.uk) }}$

We thank Eugene Harris for his comments on our article "Ontogeny and homoplasy in the papionin face" (Collard and O'Higgins 2001). In the first part of his reply, Dr. Harris argues that our study has shortcomings caused by limitations in taxon sampling. He points out that our study was restricted, for the most part, to a single species of papionin genera and, as such, did not sample variations in facial morphology within genera. Dr. Harris contends that this may be particularly problematic in the case of Macaca, which, according to some researchers, contains as many as 19 species. We agree that there is a need to test further the hypothesis that the facial similarities among Cercocebus, Lophocebus, and Macaca are plesiomorphic, whereas those among Mandrillus, Papio, and Theropithecus are homoplastic. We also agree that repeating our analyses with larger, more representative samples would be a good way of doing this. Interestingly, since submitting our article, one of us (P.O.H.) has had the opportunity to analyze samples from additional Macaca species. These analyses show that there is no significant difference in allometric trajectory between $M$. mulatta and $M$. fascicularis or between $M$. mulatta and M. sylvanus. Thus, a comparison of Cerocebus and Lophocebus with $M$. fascicularis or with $M$. sylvanus would probably yield the same result as we obtained with $M$. mulatta. Clearly, future studies may find that other macaque species differ significantly in their facial growth from M. mulatta, M. fascicularis, and M. sylvanus and also from Cercocebus and Lophocebus. However, at the moment, there is no reason to alter our conclusion that the facial similarities among Cercocebus, Lophocebus, and Macaca are plesiomorphic, whereas those between Mandrillus and Papio are homoplastic.

In the second part of his reply, Dr. Harris rejects our conclusion that our results do not support his hypothesis that facial homoplasy in Mandrillus and Papio is a consequence of selection for larger body size and strong sexual dimorphism (Harris 2000). We do not agree with Dr. Harris on this point. Our analyses of the allometric trajectories of Cercocebus, Lophocebus, Macaca, Mandrillus, and Papio show that a simple extension of ontogenetic allometry does not fully account for the homoplastic facial similarities between Mandrillus and Papio. Rather, the similarities are achieved through a combination of an extension of allometry into larger size ranges and an alteration in direction away from the ancestral allometric trajectory. Likewise, the results of our analysis of early postnatal facial morphology suggest that homoplasies are present among the papionins prior to the emergence of 
significant sexual differences in facial morphology. The corollary of this is that the homoplastic facial similarities among papionin genera (rather than between sexes of any particular genus) are unlikely to be entirely explained in terms of sexual selection. Thus, although selection for larger body size and strong sexual dimorphism may play a role in the evolution of facial homoplasy in Mandrillus and Papio, the results of our analyses clearly indicate that they are not the only processes involved.

\section{REFERENCES}

Collard, M., and O'Higgins, P. 2001. Ontogeny and homoplasy in the papionin monkey face. Evo. \& Dev. 3: 322-331.

Harris, E. E. 2000. Molecular systematics of the Old World monkey tribe Papionini: analysis of the total available genetic sequences. J. Hum. Evol. 38: 235-256. 